\title{
Assessing functional diversity: the influence of the number of the functional traits
}

Legras Gaëlle ${ }^{1,{ }^{*}}$, Loiseau Nicolas ${ }^{2,3}$, Gaertner Jean-Claude ${ }^{4}$, Poggiale J-C. ${ }^{5}$, Gaertner-Mazouni N. ${ }^{1}$

1 Univ. Polynesie FrancaisePuna'auia, French Polynesia

2 MARBEC, Univ MontpellierMontpellier, France

3 University Grenoble Alpes, CNRS, Univ. Savoie Mont Blanc, LECAGrenoble, France

${ }^{4}$ Institut de Recherche pour le Développement (IRD)Papeete, French Polynesia

${ }^{5} \mathrm{CNRS/INSU,} \mathrm{Université} \mathrm{de} \mathrm{Toulon,} \mathrm{IRD,} \mathrm{Mediterranean} \mathrm{Institute} \mathrm{of} \mathrm{Oceanography} \mathrm{(MIO)} \mathrm{UM} \mathrm{110Aix}$

Marseille UniversitéMarseille, France

* Corresponding author : Gaëlle Legras, email address : legras.gaelle@gmail.com

\begin{abstract}
:
The impact of the variation of the number of functional traits on functional diversity assessment is still poorly known. Although the covariation between these two parameters may be desirable in some situations (e.g. if adding functional traits provides relevant new functional information), it may also result from mathematical artefacts and lead to misinterpretation of the results obtained. Here, we have tested the behaviour of a set of nine indices widely used for assessing the three main components of functional diversity (i.e. functional richness, evenness and divergence), according to the variation in the number of functional traits. We found that the number of functional traits may strongly impact the values of most of the indices considered, whatever the functional information they contain. The FRic, TOP and nhypervolume indices that have been developed to characterize the functional richness component appeared to be highly sensitive to the variation in the number of traits considered. Regarding functional divergence, most of the indices considered (i.e. Q, FDis and FSpe) also showed a high degree of sensitivity to the number of traits considered. In contrast, we found that indices used to compute functional evenness (FEve and Ru), as well as one of the indices related to functional divergence (FDiv), are weakly influenced by the variation in the number of traits. All these results suggest that interpretation of most of the functional diversity indices considered cannot only be based on their values as they are, but requires taking into account the way in which they have been computed.
\end{abstract}

Keywords : Functional traits, Dissimilarity metric, Functional diversity, Index sensitivity, Trend analysis 
Functional diversity, based on the value and range of biological traits in ecosystems (Diaz \&

Cabido 2001), is becoming a major concept in ecology and ecosystem management in both the terrestrial and marine domains. An increasing body of literature suggests that functional diversity, rather than species diversity, enhances ecosystem functions such as productivity (Tilman et al. 1997, Diaz \& Cabido 2001, Hooper \& Dukes 2004, Petchey et al. 2004, Hooper et al. 2005), resilience to disturbances or invasion (Dukes 2001, Bellwood et al. 2004) and regulation in the flux of matter (Waldbusser et al. 2004, Villéger et al. 2008). It is therefore assumed that functional diversity may have the potential to link morphological, physiological and phenological variations at the individual level to ecosystem processes and patterns (Petchey et al. 2009). meta-analyses based on functional diversity aiming to understand and predict at global scale the responses of ecosystems in the face of disturbances (natural and/or anthropic, e.g. Devictor et al. 2010, Buisson et al. 2013, Stuart-Smith et al. 2013, D'agata et al. 2014, Parravicini et al. 2014, Mazel et al. 2014, Mouillot et al. 2014). The meta-analyses are generally based on the compilation of datasets which do not always share the same properties (e.g. number of species, number of functional traits or nature of functional traits; e.g. 
50 Flynn et al. 2009, Aubin et al. 2013). For example, in their study, Flynn et al. (2009) used 5 functional

51 traits for birds and 8 functional traits for plants to describe their ecological responses in a context of land

52 use intensification. In order to correctly perform these meta-analyses, it is necessary to know the influence

53 of the dataset properties on the values of the indices computed. The influence of the variation of species

54 richness, for example, has been widely studied (see Mouchet et al. 2010) and suitable methods (e.g. use of

55 nulls models, Mason et al. 2007) have been developed to counteract its effect. Although the number of

56 functional traits is also reputed to influence the index values (Podani and Schmera 2006), no studies have

57 assessed the shape (i.e. the trend) and the cause (i.e. the mathematical explanation) of this relation.

58 However, good knowledge of the mathematical relations existing between the properties of the dataset and

59 the indices used is necessary to properly perform meta-analysis.

60 In the present work, we investigated the influence of variations in the number of functional traits

61 on the basis of a large set of functional indices describing the three major components of functional

62 diversity (i.e. functional richness, functional evenness and functional divergence). We also tested the

63 impact of the dissimilarity metric used on the trend observed between the functional diversity indices and

64 the number of functional traits. The nine indices selected here are usually considered as among the most

65 promising indices for assessing and monitoring functional diversity in both marine and terrestrial

66 ecosystems (e.g. Mouillot et al. 2011, Pakeman et al. 2013, Buisson et al. 2013, Parravicini et al. 2014,

67 Fontana et al. 2015). More specifically, we have addressed the following questions: (1) To what degree

68 and how is each index affected by the number of traits considered? (2) Does the relation of each index

69 with the number of traits vary according to the metric used (Euclidean distance vs Gower dissimilarity)?

70 Finally, we regrouped the results of (1) and (2) in a table in order to help users to better interpret the results 71 they obtained.

73 Material and Methods

$74 \quad$ Selected functional indices 
75 We tested here the most promising indices used to assess functional diversity and its three major 76 components. More precisely, for assessing functional richness, which represents the amount of niche space 77 occupied by the species within a community (Mouillot et al. 2013), we included FRic (Villéger et al. 78 2008), the TOP index (Fontana et al. 2015) and the n-hypervolume index (hereafter called HYPE, Blonder 79 et al. 2014) that offer different insights into this general concept. Thus, the FRic index measures the 80 volume of functional space occupied by the species and does not consider all the species present in the 81 community for this (Villéger et al. 2008). In contrast, in considering all the species present in the 82 community, the TOP index measures more specifically the overall range and composition of functional 83 space (Fontana et al. 2015). Finally, the HYPE index represents the level of functional space filled by the 84 community (Blonder et al. 2014). In short, although these 3 indices belong to the same concept (i.e. 85 "functional richness), they do not measure exactly the same thing, and they do not have the same 86 advantages and drawbacks (see Legras et al. 2018 for more details). For the same reason, we studied the 87 functional evenness (i.e. the regularity of the distribution of the species in the functional space, Villéger et 88 al. 2008), on the basis of two complementary indices: FEve (Villéger et al. 2008) and Ru (Ricotta et al. 89 2014). The main structural difference between these two indices is that the FEve index uses the Minimum 90 Spanning Tree, MST) in its computation, which is not the case for the Ru index (Villéger et al. 2008, 91 Ricotta et al. 2014). Finally, for the functional divergence component (i.e. the proportion of total 92 abundance belonging to the most extreme functional species, Mouillot et al. 2013), we used FDiv (Villéger 93 et al. 2008), FDis (Laliberté \& Legendre 2010), Q (quadratic entropy of Rao, Botta-Dukat 2005) and FSpe 94 (Villéger et al. 2010). As for the two previous components, all indices characterizing the functional 95 divergence do not use the same mathematical tools to definite it. For example, FDis measures the mean 96 distance of species from the centroid of community, whereas Q measures the pairwise distances between 97 species (see Table 1 for further detail regarding the definitions and the mathematical formula of each 98 index). Further information about the definition, the pros and the cons of each index can be found in 99 Schleuter et al. 2010, Legras et al. 2018 and Legras \& Gaertner 2018). 
Although $\mathrm{FD}_{\mathrm{P} \& \mathrm{G}}$ (index based on dendrogram construction, Petchey \& Gaston 2002) is one of most widely used to characterize the functional richness, we have not selected this index for our study. Several studies

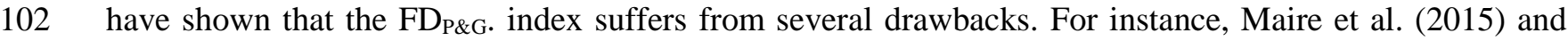

103 Loiseau et al. (2017) have demonstrated that using a dendrogram-based method could lead to functional 104 space of low quality and may alter the assessment of functional diversity and result in misleading 105 ecological conclusions.

\section{Simulated dataset}

We simulated a pool of 50 species characterized by nine functional traits. The number of functionally different species was kept constant at 50, whatever the number of traits considered, because

110 Mouchet et al. (2010) have demonstrated that this parameter could impact the functional diversity index 111 values. Abundance matrices were simulated by random selection in the log normal distribution (a common 112 pattern in nature; Preston 1948, Mouchet et al. 2010), and kept constant during the simulations. Thus, the 113 abundance matrix had 1 row and 50 columns in order to simulate the results of a sample of 50 species at a

114 single site. For the pool of 50 species, the values of traits for each species were obtained by a random 115 selection in the normal distribution $(\mu=0$ and $\sigma=1-$ see further details on this rationale in Kraft et al. 2008 116 and in Mouchet et al. 2010).

117 The method for computing the considered indices differs according to the nature (quantitative or 118 qualitative) of the functional traits (Laliberté \& Legendre, 2010). If all studied traits are quantitative and 119 no values are missing, indices are directly computed on the basis of their mathematical formula (see Table 120 1). For indices related to the functional space concept (i.e. FRic, TOP, HYPE, FEve and FDiv), the T trait 121 values observed for each species are then used as $\mathrm{T}$ coordinates for projecting each species in the 122 functional space (Villéger et al. 2008, Fontana et al. 2015). The other indices (i.e. Ru, FDis, Q and FSpe) 123 are directly computed from the dissimilarity matrix (called hereafter DMatrix). This DMatrix is obtained 124 by calculating the Euclidean distance between each pair of species. The greater the distance between two 125 species, the more these two species can be considered as functionally distinct. Conversely, if all traits are 
126 either qualitative or represent a mix of both qualitative and quantitative variables, or if they are missing 127 values, none of the indices considered can be computed, as previously explained. Regarding FRic, TOP, 128 HYPE, FEve and FDiv, species cannot be projected according to their values in qualitative functional traits 129 in a suitable manner. Similarly, for Ru, FDis, Q and FSpe, Euclidean distance cannot be used on 130 qualitative data. In these cases, authors have to use an alternative method, which differs according to the 131 indices considered. Regarding $\mathrm{Ru}, \mathrm{FDis}, \mathrm{Q}$ and FSpe, the alternative method consists in using the Gower 132 distance rather than the Euclidean distance (Laliberté \& Legendre, 2010; Podani \& Schmera 2006). For 133 FRic, TOP, HYPE, FEve and FDiv, the problem is solved by carrying out a factor analysis (a PCoA based 134 on Gower distance for the dissimilarity matrix) on the functional traits matrix (Laliberté \& Legendre, 135 2010). The factorial coordinate's matrix can then be used for projecting species on the functional space.

136 However, it is recommended by some authors to use a factor analysis (PCA or PCoA) and Gower 137 dissimilarity even if all traits are quantitative (Villéger et al. 2008, Laliberté \& Legendre 2010, Maire et al. 138 2015). In fact, this operation makes it possible to i) avoid the undesirable effect of traits correlation, and ii) 139 reduce the dimensionality of the initial dataset (a high dimensionality leading to long computation time for 140 some indices). Thus, we have chosen to apply a factor analysis in our study, even in this case, in order to 141 be closer to the situations encountered in the case studies. For the sake of brevity, only results with the 142 conservation of the two first factorial coordinates are presented here (we also simulated situations with 4 143 and 6 factorial coordinates that provided similar results, see Supplementary Material, Fig. S1). To test the 144 impact of the metric used on the relation between functional index values and the number of functional 145 traits, Euclidean vs Gower metrics (respectively hereafter called 'Euclidean-based Approach' and 146 'Gower-based Approach') were applied on the simulated data to compute DMatrix. To test the influence of 147 the number of functional traits, we computed the functional indices for the number of traits varying from 148 two to nine.

149 All these simulations were performed 999 times using the R software (R Development Core Team 2014) 150 through FD package (Laliberté \& Legendre 2010), TOP.index function (Fontana et al. 2015), FeveR 151 function (Ricotta et al. 2014) and fspe function (Villéger et al. 2010). Moreover, it is important to note that 
152 due to our simulation procedure (i.e. functional traits were randomly selected and bootstrapped), results 153 obtained remained independent of trait identity (i.e. they are not dependent on the functional information 154 contained by traits). More specifically, this simulation procedure enables us to strictly evaluate the 155 mathematical relationship (i.e. the trend) between the functional indices and the number of functional 156 traits, whatever the functional information they contain. The relation between the functional index values 157 and the number of functional traits for both metrics was tested by means of a trend analysis in an ANOVA 158 framework.

160 Results

\section{Euclidean-based Approach}

162 When using the Euclidean-based approach, the three indices characterizing functional richness (i.e. FRic, 163 TOP and HYPE indices) are influenced by the number of traits (t) used. More specifically, these indices 164 increased with the number of functional traits and are characterized by a linear relationship for FRic and 165 the TOP index (Table 2, adjusted R-squared $\left(\operatorname{adj} . \mathrm{R}^{2}\right)=0.74$ and 0.61 respectively), and a quartic 166 relationship for the HYPE index $\left(\operatorname{adj} . \mathrm{R}^{2}=0.85\right)$. For the evenness component, FEve and Ru remained stable 167 relative to the number of functional traits (Fig 2), despite highlighting a cubic trend observed for the Ru 168 index (Table 2, adj. $R^{2}$ for $R u$ index $=0.93$ ). In contrast, for the divergence component, only the FDiv 169 index was not influenced by the number of functional traits $\left(\operatorname{adj} \cdot R^{2}\right.$ close to 0 and $p$-value $=0.17$, Table 2$)$.

170 The three other indices (i.e. FDis, FSpe and Q) were both positively and closely correlated with the number of functional traits (Fig 2). Furthermore, these indices are mainly characterized by a cubic 172 relationship with the number of functional traits for the FDis and FSpe indices (Table 2, both adj. $\mathrm{R}^{2}=0.99$ ), 173 and by a linear relationship for the $\mathrm{Q}$ index (Table 2 , adj. $\mathrm{R}^{2}=1$ ). We note that indices projected into 174 functional space (i.e. FRic, TOP, HYPE, FEve and FDiv) showed the same trends whatever the number of 175 dimensions of functional space (see Supplementary Material - Fig. S1). 
The FRic, TOP and HYPE indices strongly decreased with the increasing number of functional traits in a 2-D functional space (Fig 3). This trend is observed whatever the number of dimensions considered for the functional space (i.e. for 4 and 6 dimensions, see Supplementary Material - Fig. S2.). The FRic and TOP indices are respectively characterized by a significant cubic relationship with the number of functional traits (see Table 2, adj. $\mathrm{R}^{2}=0.95$ and 0.93 respectively), whereas the HYPE index is characterized by a quartic relationship (adj. $\left.\mathrm{R}^{2}=0.96\right)$. For the evenness component, the number of functional traits very weakly influences both indices (i.e. FEve and Ru indices), as demonstrated by the trend analysis (Table 2, $\operatorname{adj} \cdot \mathrm{R}^{2}=0.01$ for FEve index and adj. $\mathrm{R}^{2}=0.02$ for $\mathrm{Ru}$ index). For the divergence component, we also observed low adj. $\mathrm{R}^{2}$ for FDiv index (Table 2, adj. $\mathrm{R}^{2}=0.02$ ). Conversely, FDis, FSpe and Q decreased with the number of functional traits and they are characterized by a significant quartic relationship (see Table 2, $\operatorname{adj} . \mathrm{R}^{2}=0.82$ for both FDis and FSpe and 0.78 for Q).This result implies that the number of functional traits strongly affects these three indices values when the Gower dissimilarity is used.

\section{Discussion}

The choice of traits used is of primary importance for assessing functional diversity because it is dependent on both the ecological question addressed and the characteristics of the community studied (Petchey \& Gaston 2006). Here, we present the first study strictly focused on the relation between the functional diversity indices and the number of functional traits according to the dissimilarity metrics used. Although the number of functional entities (i.e. species sharing the same combination of functional trait values) remains constant, our results showed that functional diversity indices values are influenced by the variations in the number of traits (independently of the functional information they contain) and by the dissimilarity metric used (Euclidean distance or Gower dissimilarity).

In our study, functional richness is investigated on the basis of the FRic, TOP and HYPE indices. These three indices are all based on the construction of a geometrical objet in a multidimensional space: the 
convex hull for the FRic and TOP indices (Villéger et al. 2008, Fontana et al. 2015) and the hypervolume for the HYPE index (Blonder et al. 2014). Here, we showed that these three indices strongly increased with the number of functional traits with Euclidean distance, while they decreased with Gower dissimilarity. This is due to the fact that the Euclidean distance is not normed and positively correlated with the number of functional traits (Mason et al. 2005, Podani \& Schmera 2006, Mason et al. 2007). Using Euclidean distance, the more functional traits there are, the greater is the distance between species and the more widely spaced in the functional space the species are. Thus, the surface and volume of the geometric object built (i.e. convex hull or hypervolume) are greater and these three indices (i.e. FRic, TOP 213 dissimilarity is explained by the fact that the distance between two points A and B decreases with the 214 increase in the number of functional traits after projection in the n-dimensional space when we use Gower 215 dissimilarity (with n constant, see Supplementary Material - Fig S3).

216 Regarding functional evenness, both indices (FEve and Ru) are weakly influenced by the number of 217 functional traits, whatever the distance metric used. In addition, FEve and Ru are not - or only weakly 218 influenced by the species richness (Mouchet et al. 2010, Ricotta et al. 2014). Choosing between FEve and $219 \mathrm{Ru}$ should depend on the information sought by the user. FEve is focused on the regularity of the distances 220 between each species (or individuals) and its nearest neighbour, weighted by their relative abundance 221 (Villéger et al. 2008), whereas the Ru index measures the regularity of the distances between each species 222 (or individuals) and all the remaining species (or individuals) of the community, weighted by their relative 223 abundance (Ricotta et al. 2014).

224 Finally, functional divergence represents how spread-out or how clumped the species are in the niche space, weighted by the relative abundance (Mason et al. 2005), and is usually represented by FDiv, FDis and Q (Pavoine \& Bonsall 2011). In addition, the aim of the FSpe index (i.e. quantifying how far apart the 227 species are from the centre of gravity of the species pool, Villéger et al. 2010, Pla et al. 2012) suggests that this index can also be used for assessing this component. Our results showed that the FDiv index is weakly 
230 Euclidean distance or Gower dissimilarity). In contrast, our work has demonstrated that FDis, FSpe and Q 231 were positively correlated with the number of functional traits when we used Euclidean distance, whereas 232 they are negatively correlated with this parameter when we used Gower dissimilarity. FDis, FSpe and Q 233 being mathematically proportional to the dissimilarity metric (cf. Table 1), the patterns we observed for 234 these indices are directly derived from the relation existing between the dissimilarity metric used and the 235 number of functional traits (i.e. positive relationship for Euclidean metric (Podani \& Schmera 2006) and 236 negative relationship for Gower dissimilarity, see Supplementary Material - Fig S3.)

The selection of indices to be used for properly assessing the main facets of functional diversity of a given community is a complex issue (Loiseau \& Gaertner 2015). It primarily depends on the aims of each study and the nature of the available data. With this choice is associated the choice of both the identity and the number of traits to select. Although of primordial importance, this choice could be difficult because it 242 implies very good knowledge of (i) the level of implication of each functional trait in the role of the 243 species in ecosystem, and (ii) the number of functional traits to select to correctly describe a given 244 function. For example, for the food acquisition and locomotion of fishes, Villéger et al. (2010) suggested 245 using 16 morphometric traits to take into account these functions. In another study, Mouillot et al. (2014) 246 used only six functional traits (with only one related to morphometry) to describe the same two functions. 247 This example illustrates that the choice of the number of functional traits to be used for describing a given 248 function is not universal (even for identical species), but based on a partly subjective rationale (Hortal et 249 al. 2015, De Bello et al. 2017). Moreover, this point is particularly difficult to deal with because it gives 250 rise to the dilemma of choosing between "select enough traits to describe a given function" and "select too 251 many traits for a given function", that could generate an overestimation of this function compared to the 252 other functions (overestimation generated by a trivial correlation between traits). A method of ordination 253 (e.g. PCA or PCoA) will avoid these correlations, but ordination entails the risk of a loss of information 254 (since the ordination axes can only capture a proportion of the variation in functional trait values across 255 species, Villéger et al. 2008). 
256 Furthermore, series of sensitivity can be applied to test the robustness of values of functional diversity 257 indices against the number (and the identity) of functional traits (see Mouillot et al. 2014). This can be 258 done by rerunning analyses using all combinations of $(n-1)$ traits out of $n$. The results obtained might serve 259 as a basis for the differentiation of the effects due to ecological phenomena from those due to the 260 mathematical artefacts. An alternative solution to overcome this sensitivity might be to compare 261 beforehand the values of indices to null models respecting the same conditions as those used to compute 262 the indices (e.g. same number of functional traits, same dissimilarity metric used). To date, the use of null 263 models has been recommended by several authors (Mason et al. 2007, Mason et al. 2008, Villéger et al. $2642008,2010)$ to get around the problem of the sensitivity of these indices to the variation of species richness 265 (Mouchet et al. 2010). With the aim of helping ecologists to build proper null models, we have 266 summarized in Table 3 the sensitivity to these factors of the most widely used functional diversity indices 267 on the basis of the results of our study.

268 To sum up, three operations can be envisaged once the best set of functional traits has been chosen by the 269 users according to the aim of the study. Firstly, a series of sensitivities tests can be applied on the set of 270 traits to avoid some misinterpretation due to the mathematical artefacts. Secondly, a factor analysis (as 271 PCA or PCoA) can be performed to reduce the number of dimensions of functional space. For some 272 indices, a large number of dimensions can entail extensive computation time. Thirdly, to get around the 273 sensitivity of indices to the data used for their computation (e.g. number of functional traits, dissimilarity 274 metric used, species richness), we can compare the value of the index to the values obtained under null 275 models.

276 Our study is of primarily theoretical importance in providing a better understanding of the mathematical 277 properties of functional diversity indices that may impact the interpretation of results obtained by the 278 ecologists in the field. For example, our study highlights the fact that is highly hazardous to compare the 279 functional diversity values of i) studies which do not exactly share the same number of functional traits, or 280 ii) studies in which functional diversity values are not computed with the same dissimilarity metric. By 
281 improving and clarifying the theoretical properties of these indices, our study could help ecologists to 282 better estimate both the diversity patterns and the role of the taxa studied in ecosystem functioning.

284 Finally, many studies have recently focused on the development of new functional diversity indices 285 (Villéger et al. 2008, Laliberté \& Legendre 2010, Fontana et al. 2015). While this domain of research is 286 necessary, our study strongly supports the need to devote a significant methodological effort to the 287 assessment of the mathematical properties of indices already developed in order to better assess their limits 288 and constraints. This would be not only a major step towards avoiding any misinterpretation of the results 289 obtained by the users, but would also provide a methodological basis for orientating future research 290 focused on the development of new indices.

\section{Acknowledgements}

293 This work, and in particular the PhD fellowship of G.L., was supported by the University of French 294 Polynesia (ED 469) and Labex CORAIL and funded by the Research Ministry of French Polynesia 295 (Délégation à la Recherche de Polynesie Française). We are grateful to M. Paul for corrections of the 296 English. We declare that no competing interests exist. 
Aubin, I., Venier, L., Pearce, J., \& Moretti, M. (2013). Can a trait-based multi-taxa approach improve our assessment of forest management impact on biodiversity?. Biodiversity and conservation, 22(12), 2957-2975.

Bellwood, D.R., Hughes, T.P., Folke, C., and Nyström, M. (2004). Confronting the coral reef crisis. Nature 429, 827-833.

Blonder, B., Lamanna, C., Violle, C., \& Enquist, B. J. (2014). The n-dimensional hypervolume. Global Ecology and Biogeography, 23(5), 595-609.

Botta-Dukát, Z. (2005). Rao's quadratic entropy as a measure of functional diversity based on multiple traits. Journal of Vegetation Science 16, 533-540.

Buisson, L., Grenouillet, G., Villéger, S., Canal, J., and Laffaille, P. (2013). Toward a loss of functional diversity in stream fish assemblages under climate change. Global Change Biology 19, 387-400.

D’agata, S., Mouillot, D., Kulbicki, M., Andréfouët, S., Bellwood, D. R., Cinner, J. E., ... \& Vigliola, L. (2014). Human-mediated loss of phylogenetic and functional diversity in coral reef fishes. Current Biology, 24(5), 555-560.

De Bello, F., Šmilauer, P., Diniz-Filho, J. A. F., P Carmona, C., Lososová, Z., Herben, T., \& Götzenberger, L. (2017). Decoupling phylogenetic and functional diversity to reveal hidden signals in community assembly. Methods in Ecology and Evolution. DOI: 10.1111/2041-210X.12735

Devictor, V., Mouillot, D., Meynard, C., Jiguet, F., Thuiller, W., \& Mouquet, N. (2010). Spatial mismatch and congruence between taxonomic, phylogenetic and functional diversity: the need for integrative conservation strategies in a changing world. Ecology letters, 13(8), 1030-1040.

Diaz, S., and Cabido, M. (2001). Vive la difference: plant functional diversity matters to ecosystem processes. Trends Ecol. Evol. 16, 646-655.

Dukes, J.S. (2001). Productivity and complementarity in grassland microcosms of varying diversity. Oikos $94,468-480$. 
Flynn, D. F., Gogol-Prokurat, M., Nogeire, T., Molinari, N., Richers, B. T., Lin, B. B., ... \& DeClerck, F. (2009). Loss of functional diversity under land use intensification across multiple taxa. Ecology letters, 12(1), 22-33.

Fontana, S., Petchey, O. L., \& Pomati, F. (2015). Individual-level trait diversity concepts and indices to comprehensively describe community change in multidimensional trait space. Functional Ecology. doi: 10.1111/1365-2435.12551

Hooper D. U., Chapin III F. S., Ewel J. J., Hector A., Inchausti P., Lavorel S., Lawton J. H., Lodge D. M., Loreau M., Naeem S., Schmid B., Setälä H., Symstad A. J., Vandermeer J., and Wardle D. A. (2005). Effects of biodiversity on ecosystem functioning: a consensus of current knowledge. Ecological Monographs 75:3-35

Hooper, D.U., and Dukes, J.S. (2004). Overyielding among plant functional groups in a long-term experiment. Ecol. Lett. 7, 95-105.

Hortal, J., de Bello, F., Diniz-Filho, J. A. F., Lewinsohn, T. M., Lobo, J. M., \& Ladle, R. J. (2015). Seven shortfalls that beset large-scale knowledge of biodiversity. Annual Review of Ecology, Evolution, and Systematics, 46, 523-549.

Kraft, N.J., Valencia, R., and Ackerly, D.D. (2008). Functional traits and niche-based tree community assembly in an Amazonian forest. Science 322, 580-582.

Laliberté, E., and Legendre, P. (2010). A distance-based framework for measuring functional diversity from multiple traits. Ecology 91, 299-305.

Legras. G., \& Gaertner, J.C. (2018). Assessing functional evenness with the FEve index: A word of warning. Ecological Indicators, 90, 257-260.

Legras, G., Loiseau, N., \& Gaertner, J. C. (2018). Functional richness: Overview of indices and underlying concepts. Acta Oecologica, 87, 34-44.

Loiseau, N., \& Gaertner, J. C. (2015). Indices for assessing coral reef fish biodiversity: the need for a change in habits. Ecology and evolution, 5(18), 4018-4027. 
Loiseau, N., Legras, G., Gaertner, J. C., Verley, P., Chabanet, P., \& Mérigot, B. (2017). Performance of partitioning functional beta-diversity indices: Influence of functional representation and partitioning methods. Global Ecology and Biogeography, 26(6), 753-762.

Maire, E., Grenouillet, G., Brosse, S., and Villéger, S. (2015). How many dimensions are needed to accurately assess functional diversity? a pragmatic approach for assessing the quality of functional spaces. Global Ecology and Biogeography.

Mason, N.W., Irz, P., Lanoiselée, C., Mouillot, D., and Argillier, C. (2008). Evidence that niche specialization explains species-energy relationships in lake fish communities. Journal of Animal Ecology 77, 285-296.

Mason, N.W., Lanoiselée, C., Mouillot, D., Irz, P., and Argillier, C. (2007). Functional characters combined with null models reveal inconsistency in mechanisms of species turnover in lacustrine fish communities. Oecologia 153, 441-452.

Mason, N.W., Mouillot, D., Lee, W.G., and Wilson, J.B. (2005). Functional richness, functional evenness and functional divergence: the primary components of functional diversity. Oikos 111, 112-118.

Mazel, F., Guilhaumon, F., Mouquet, N., Devictor, V., Gravel, D., Renaud, J., ... \& Thuiller, W. (2014). Multifaceted diversity-area relationships reveal global hotspots of mammalian species, trait and lineage diversity. Global ecology and biogeography, 23(8), 836-847.

Mouchet, M.A., Villeger, S., Mason, N.W., and Mouillot, D. (2010). Functional diversity measures: an overview of their redundancy and their ability to discriminate community assembly rules. Functional Ecology 24, 867-876.

Mouillot, D., Villéger, S., Parravicini, V., Kulbicki, M., Arias-González, J.E., Bender, M., Chabanet, P., Floeter, S.R., Friedlander, A., Vigliola, L., et al. (2014). Functional over-redundancy and high functional vulnerability in global fish faunas on tropical reefs. Proceedings of the National Academy of Sciences 111, 13757-13762.

Mouillot, D., Villéger, S., Scherer-Lorenzen, M., \& Mason, N. W. (2011). Functional structure of biological communities predicts ecosystem multifunctionality. PloS one, 6(3), e17476. 
Pakeman, R.J., and Eastwood, A. (2013). Shifts in functional traits and functional diversity between vegetation and seed bank. Journal of Vegetation Science 24, 865-876.

Parravicini, V., Villéger, S., McClanahan, T.R., Arias-González, J.E., Bellwood, D.R., Belmaker, J., Chabanet, P., Floeter, S.R., Friedlander, A.M., Guilhaumon, F., et al. (2014). Global mismatch between species richness and vulnerability of reef fish assemblages. Ecology Letters 17, 11011110.

Pavoine, S., and Bonsall, M.B. (2011). Measuring biodiversity to explain community assembly: a unified approach. Biological Reviews 86, 792-812.

Petchey, O. L., \& Gaston, K. J. (2002). Functional diversity (FD), species richness and community composition. Ecology Letters, 5, 402-411.

Petchey, O.L., and Gaston, K.J. (2006). Functional diversity: back to basics and looking forward. Ecology Letters 9, 741-758.

Petchey, O.L., Hector, A., and Gaston, K.J. (2004). How do different measures of functional diversity perform? Ecology 85, 847-857.

Petchey, O.L., O’Gorman, E.J., and Flynn, D.F. (2009). A functional guide to functional diversity measures. Biodiversity, Ecosystem Functioning, and Human Wellbeing An Ecological and Economic Perspective 49-60.

Pla, L., Casanoves, F., and Di Rienzo, J. (2012). Functional diversity indices. In Quantifying Functional Biodiversity, (Springer), pp. 27-51.

Podani, J., and Schmera, D. (2006). On dendrogram-based measures of functional diversity. Oikos, 115, $179-185$.

Preston, F. W. (1948). The commonness, and rarity, of species. Ecology, 29,254-283.

Ricotta, C., Bacaro, G., \& Moretti, M. (2014). A new measure of functional evenness and some of its properties. PloS one, 9, e104060.

Schleuter, D., Daufresne, M., Massol, F., \& Argillier, C. (2010). A user's guide to functional diversity indices. Ecological Monographs, 80(3), 469-484. 
400 Stuart-Smith, R. D., Bates, A. E., Lefcheck, J. S., Duffy, J. E., Baker, S. C., Thomson, R. J., ... \& Becerro, 401 M. A. (2013). Integrating abundance and functional traits reveals new global hotspots of fish 402 diversity. Nature, 501(7468), 539.

403 Tilman, D., Naeem, S., Knops, J., Reich, P., Siemann, E., Wedin, D., Ritchie, M., and Lawton, J. (1997). 404 Biodiversity and ecosystem properties. Science 278, 1866-1867.

405 Villéger, S., Mason, N.W.H., and Mouillot, D. (2008). New multidimensional functional diversity indices 406 for a multifaceted framework in functional ecology. Ecology 89, 2290-2301.

407 Villéger, S., Miranda, J.R., Hernández, D.F., and Mouillot, D. (2010). Contrasting changes in taxonomic 408 vs. functional diversity of tropical fish communities after habitat degradation. Ecological $409 \quad$ Applications 20, 1512-1522.

410 Waldbusser, G.G., Marinelli, R.L., Whitlatch, R.B., and Visscher, P.T. (2004). The effects of infaunal 411 biodiversity on biogeochemistry of coastal marine sediments. Limnology and Oceanography 49, $412 \quad 1482-1492$. 
Table 1: Functional diversity measures. From Mouchet et al. 2010, modified.

\begin{tabular}{|c|c|c|c|c|c|}
\hline $\begin{array}{l}\text { Functional } \\
\text { diversity } \\
\text { Component }\end{array}$ & $\begin{array}{l}\text { Functional } \\
\text { diversity } \\
\text { Index }\end{array}$ & Authors & Mathematical formula & Description & Data needed \\
\hline \multirow{3}{*}{ 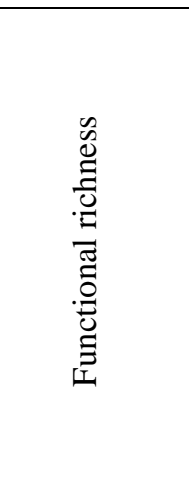 } & TOP & Fontana et al. 2015 & Quickhull algorithm & $\begin{array}{l}\text { Sum of successive convex } \\
\text { hull area. Density of } \\
\text { functional space filled by the } \\
\text { community. }\end{array}$ & $\begin{array}{l}\text { Trait values or factorial } \\
\text { coordinates }\end{array}$ \\
\hline & FRic & Villéger et al. 2008 & Quickhull algorithm & $\begin{array}{l}\text { Convex Hull Volume. } \\
\text { Amount of functional space } \\
\text { filled by the community. }\end{array}$ & $\begin{array}{l}\text { Trait values or factorial } \\
\text { coordinates }\end{array}$ \\
\hline & HYPE & Blonder et al. 2014 & Computation of n-hypervolume & $\begin{array}{l}\text { Level of functional } \\
\text { space filled by the } \\
\text { community }\end{array}$ & $\begin{array}{l}\text { Trait values or factorial } \\
\text { coordinates }\end{array}$ \\
\hline \multirow{2}{*}{ 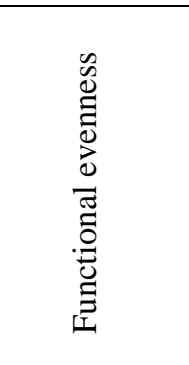 } & FEve & Villéger et al. 2008 & $F E v e=\frac{\sum_{l=1}^{S-1} \min \left(P E W_{l}, \frac{1}{S-1}\right)-\left(\frac{1}{S-1}\right)}{1-\frac{1}{S-1}}$ & $\begin{array}{l}\text { Sum of MST branch length } \\
\text { (l) weighted by relative } \\
\text { abundance. }\end{array}$ & $\begin{array}{l}\text { Trait values or factorial } \\
\text { coordinates }\end{array}$ \\
\hline & $\mathrm{Ru}$ & Ricotta et al. 2014 & $R u=\frac{\sum_{i}^{S} \min \left\{\left[\frac{\left(p_{i} * \sum_{j \neq i}^{S} \frac{p_{j}}{1-p_{i}} * d_{i j}\right)}{\sum_{i}^{S} p_{i} \sum_{j \neq i}^{S} \frac{p_{j}}{1-p_{i}}} * d_{i j}, \frac{1}{S}\right]\right\}-1 / s}{1-1 / S}$ & $\begin{array}{l}\text { Regularity with which } \\
\text { species are distributed along } \\
\text { the tree, together with the } \\
\text { evenness in their abundances }\end{array}$ & $\begin{array}{l}\text { Trait values or factorial } \\
\text { coordinates }\end{array}$ \\
\hline \multirow{2}{*}{ 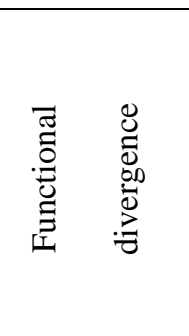 } & FDiv & Villéger et al. 2008 & $F D i v=\frac{\Delta d+\overline{d G}}{\Delta|d|+d G}$ & $\begin{array}{l}\text { Species deviance from the } \\
\text { mean distance to the center } \\
\text { of gravity weighted by } \\
\text { relative abundance. }\end{array}$ & $\begin{array}{l}\text { Trait values or factorial } \\
\text { coordinates }\end{array}$ \\
\hline & FDis & $\begin{array}{l}\text { Laliberté \& } \\
\text { Legendre } 2010\end{array}$ & $F D i s=\frac{\sum p_{i} z_{i}}{\sum p_{i}}$ & $\begin{array}{l}\text { Mean distance of individual } \\
\text { species to the centroid of all } \\
\text { species to the community. }\end{array}$ & Dissimilarity matrix \\
\hline
\end{tabular}




\begin{tabular}{|l|l|l|l|l|l}
\hline & Q & $\begin{array}{l}\text { Rao (1982) \& } \\
\text { Botta-Dukat (2005) }\end{array}$ & $Q=\sum_{i=1}^{S} \sum_{j=1}^{S} p_{i} p_{j} d_{i j}$ & $\begin{array}{l}\text { Sum of pairwise distances } \\
\text { between species weighted by } \\
\text { relative abundance. }\end{array}$ & $\begin{array}{l}\text { Dissimilarity matrix } \\
\text { FSpe }\end{array}$ \\
\cline { 2 - 5 } & Villéger et al. 2010 & FSpe $=\sum_{i=1}^{S}\left(p_{i} * d G_{i}\right)$ & $\begin{array}{l}\text { Euclidean distance of } \\
\text { species to the center of } \\
\text { gravity of all the species } \\
\text { weighted by relative } \\
\text { abundance. }\end{array}$ \\
\hline
\end{tabular}

$d_{i j}$ : dissimilarity between species $i$ and $j . S:$ total species richness. $p_{i}$ : relative abundance of species $i . d G:$ mean distance of species to the center of gravity. $\Delta d:$ sum of abundance-weighted deviances. $\Delta|d|$ : absolute abundance-weighted deviances from the center of gravity (see Villéger et al. 2008 for more explanations about computations of $d G, \Delta d$ and $\Delta|d|$ ) . $P E W_{l}:$ partial weighted evenness

$\left(P E W_{l}={ }^{\frac{\operatorname{dist}(i, j)}{w_{i}+w_{j}}} / \sum_{l=1}^{S-1} \frac{\operatorname{dist}(i, j)}{w_{i}+w_{j}}\right.$, for each branch $l$ of the MST) $z_{i}$ : distance of individual species to the centroid of the community. 
Table 2: Relationship observed between functional diversity indices and number of functional traits by a trend analysis in an ANOVA framework when Euclidean distance or Gower dissimilarity is used for index computation. Code, formula and a short description of each functional diversity index is given in Table 1.

\begin{tabular}{|c|c|c|c|}
\hline $\begin{array}{c}\text { Functional diversity } \\
\text { index }\end{array}$ & Type of relation & p-value associated & Adjusted R-squared \\
\hline & \multicolumn{3}{|c|}{ using Euclidean distance } \\
\hline FRic & Linear & $<2,2 \mathrm{e}-16$ & 0,74 \\
\hline TOP & Linear & $<2,2 \mathrm{e}-16$ & 0,61 \\
\hline HYPE & Quartic & $<2,2 \mathrm{e}-16$ & 0,85 \\
\hline FEve & Quadratic & 0,04 & 0,003 \\
\hline $\mathrm{Ru}$ & Cubic & $<2,2 \mathrm{e}-16$ & 0,93 \\
\hline FDiv & Quadratic & 0,17 & 0,001 \\
\hline FDis & Cubic & $<2,2 \mathrm{e}-16$ & 0,99 \\
\hline FSpe & Cubic & $<2,2 \mathrm{e}-16$ & 0,99 \\
\hline \multirow[t]{2}{*}{ Q } & Linear & $<2,2 \mathrm{e}-16$ & 1 \\
\hline & \multicolumn{3}{|c|}{ using Gower dissimilarity } \\
\hline FRic & Cubic & $<2,2 \mathrm{e}-16$ & 0,95 \\
\hline TOP & Cubic & $<2,2 \mathrm{e}-16$ & 0,93 \\
\hline HYPE & Quartic & $<2,2 \mathrm{e}-16$ & 0,96 \\
\hline FEve & Linear & 0,002 & 0,01 \\
\hline $\mathrm{Ru}$ & linear & $3,57 e-05$ & 0,02 \\
\hline FDiv & Quadratic & 0,001 & 0,02 \\
\hline FDis & Quartic & 4,67e-05 & 0.82 \\
\hline FSpe & Quartic & $5,02 e-05$ & 0,82 \\
\hline Q & Quartic & $2 \mathrm{e}-07$ & 0,78 \\
\hline
\end{tabular}


Table 3: Sensitivity of functional diversity indices to the main factors of variation between different datasets. Mathematical formulas of indices are given in Table 1. For the number of traits factor (between 2 to 9), we tested whether the functional diversity values remain the same (insensitive) or not (sensitive) with the increase in this factor. For the dissimilarity metric used (Euclidean vs Gower), we tested whether the patterns of functional diversity indices between the two datasets remain the same (insensitive) or not (sensitive) according to trend analysis in an ANOVA framework $(\alpha=0.05)$.

\begin{tabular}{|c|c|c|c|}
\hline \multirow[b]{2}{*}{$\begin{array}{l}\text { Functional } \\
\text { diversity } \\
\text { component }\end{array}$} & \multirow[b]{2}{*}{$\begin{array}{l}\text { Functional } \\
\text { diversity } \\
\text { index }\end{array}$} & \multicolumn{2}{|c|}{ Sensitivity to... } \\
\hline & & Number of traits & Distance metric used \\
\hline \multirow{3}{*}{ 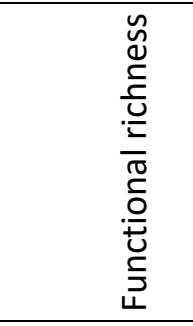 } & FRic & Yes & Yes \\
\hline & TOP & Yes & Yes \\
\hline & HYPE & Yes & Yes \\
\hline \multirow{2}{*}{ 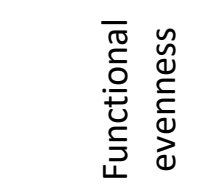 } & FEve & No & No \\
\hline & Ru & No & No \\
\hline \multirow{4}{*}{ 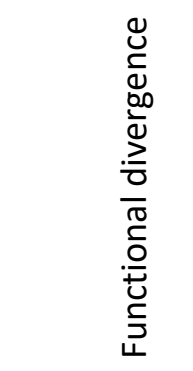 } & FDiv & No & No \\
\hline & FDis & Yes & Yes \\
\hline & FSpe & Yes & Yes \\
\hline & $Q$ & Yes & Yes \\
\hline
\end{tabular}




\section{Figures}

Fig 1: General framework to compute the nine functional diversity indices studied through the two approaches considered in this study. Modified from Villéger et al. 2008. $\operatorname{Tr}_{\mathrm{i}}=\mathrm{i}^{\text {th }}$ functional trait, $\mathrm{Sp} \mathrm{j}=\mathrm{j}^{\text {th }}$ species. $\mathrm{PC}=$ Principal component (resulting from a PCoA or a PCA). Comm = Community. Code, formula and a short description of each functional diversity index is given in Table 1 .

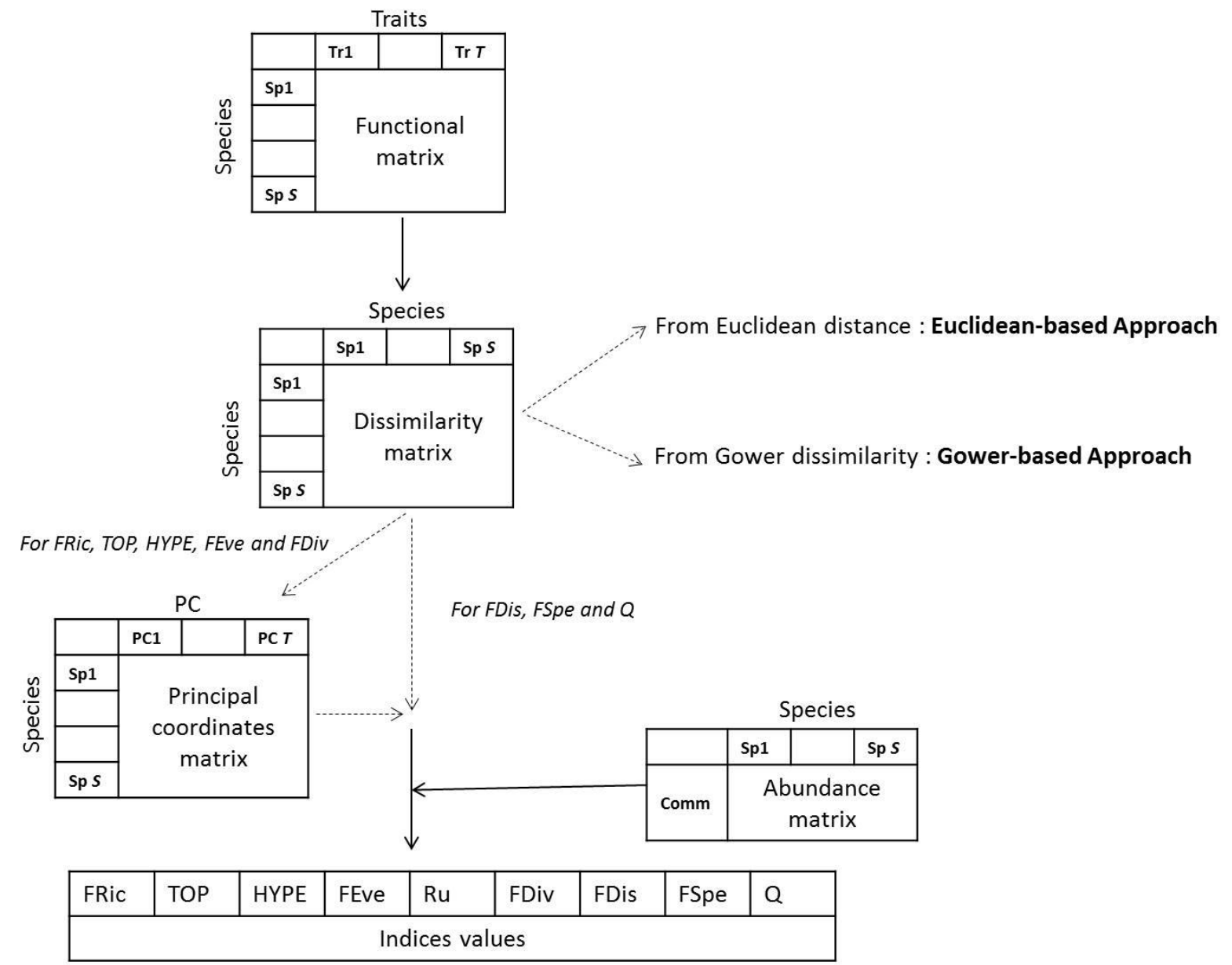


Fig 2: Influence of the number of functional traits on the estimation of functional diversity indices with the Euclidean-based Approach (i.e. computing functional indices from the Euclidean distance matrix). Curves are mean values of indices and vertical bars, associated standard errors. Code, formula and a short description of each functional diversity index is given in Table 1.
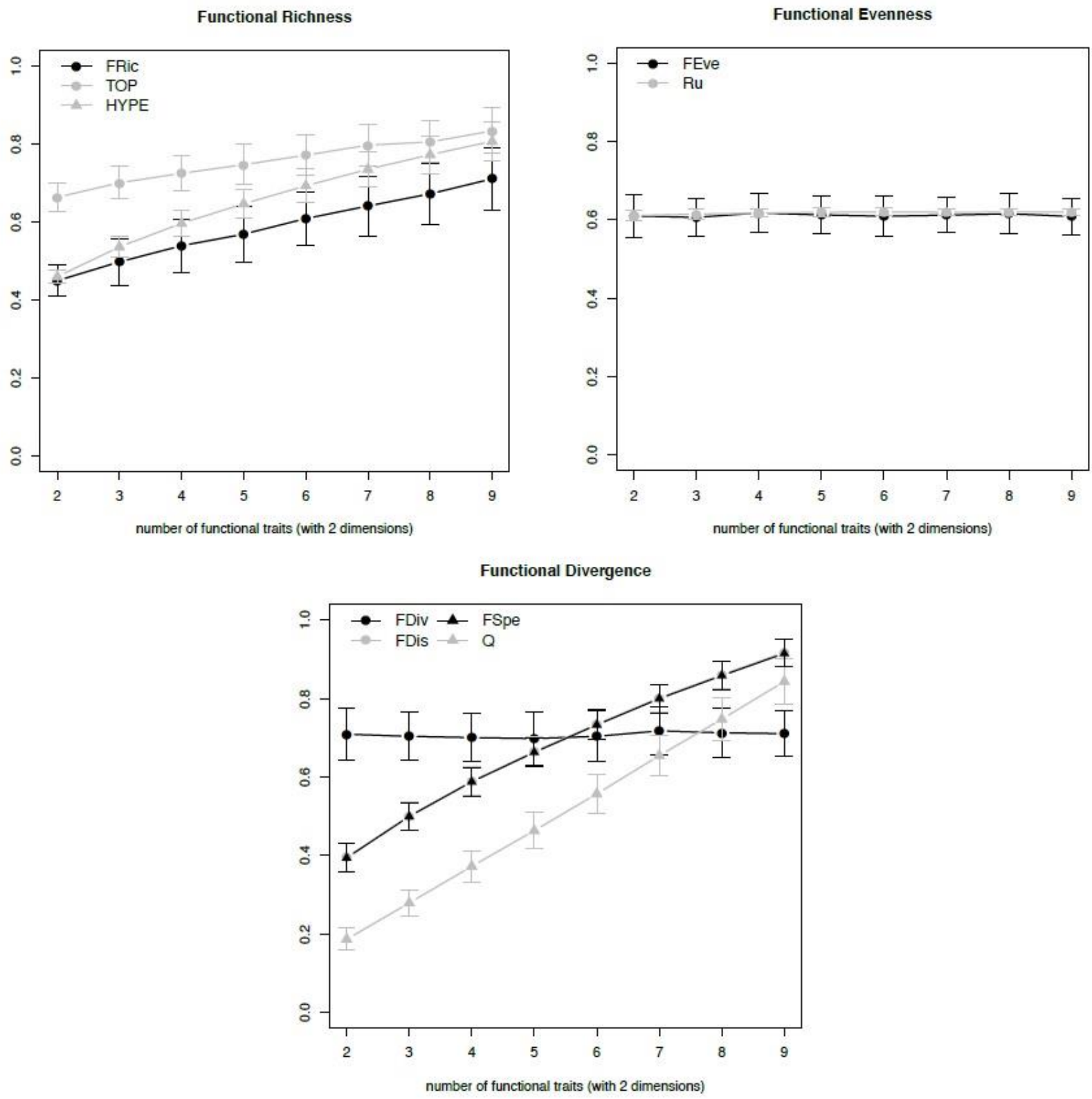
Fig 3: Influence of the number of functional traits on the estimation of functional diversity indices with a Gower-based Approach (i.e. computing functional indices from the Gower dissimilarity matrix). Curves are mean values of indices and vertical bars, associated standard errors. Code, formula and a short description of each functional diversity index is given in Table 1.
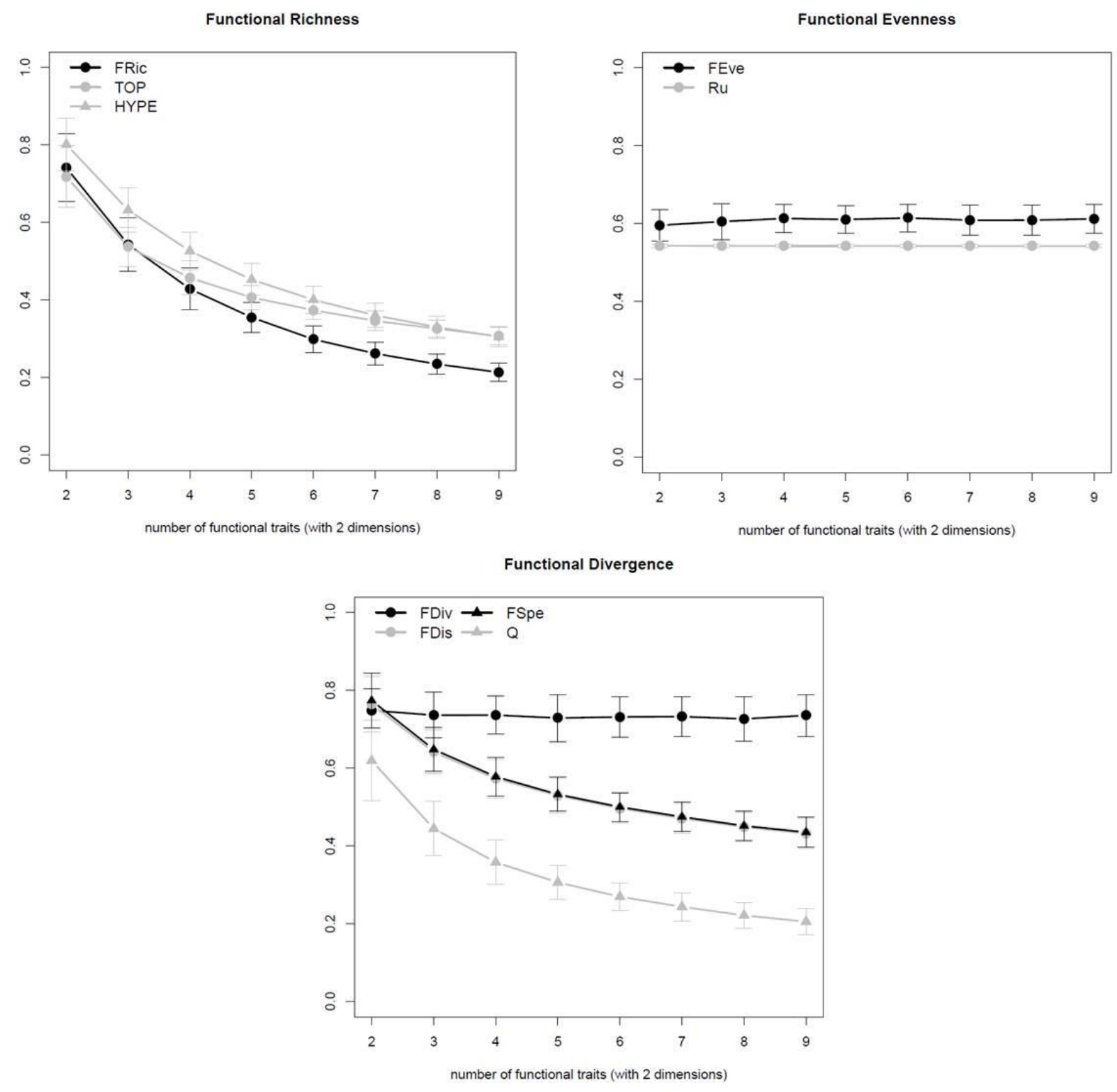